\title{
A tabu search heuristic for the Equitable Coloring Problem
}

\author{
I. Méndez Díaz ${ }^{3}$, G. Nasini ${ }^{1,2}$ and D. Severín ${ }^{1,2}$ \\ 1 Facultad de Ciencias Exactas, Ingeniería y Agrimensura \\ Universidad Nacional de Rosario, Argentina \\ \{nasini,daniel\}@fceia.unr.edu.ar \\ 2 CONICET, Argentina \\ 3 Facultad de Ciencias Exactas y Naturales \\ Universidad de Buenos Aires, Argentina \\ imendez@dc.uba.ar
}

\begin{abstract}
The Equitable Coloring Problem is a variant of the Graph Coloring Problem where the sizes of two arbitrary color classes differ in at most one unit. This additional condition, called equity constraints, arises naturally in several applications. Due to the hardness of the problem, current exact algorithms can not solve large-sized instances. Such instances must be addressed only via heuristic methods.

In this paper we present a tabu search heuristic for the Equitable Coloring Problem. This algorithm is an adaptation of the dynamic TABUCOL version of Galinier and Hao. In order to satisfy equity constraints, new local search criteria are given.

Computational experiments are carried out in order to find the best combination of parameters involved in the dynamic tenure of the heuristic. Finally, we show the good performance of our heuristic over known benchmark instances.
\end{abstract}

Keywords: equitable coloring $\cdot$ tabu search $\cdot$ combinatorial optimization

\section{Introduction}

The Graph Coloring Problem (GCP) is a very well-studied $\mathcal{N} \mathcal{P}$-Hard problem since it models many applications such as scheduling, timetabling, electronic bandwidth allocation and sequencing problems.

Given a simple graph $G=(V, E)$, where $V$ is the set of vertices and $E$ is the set of edges, a $k$-coloring of $G$ is a partition of $V$ into $k$ sets $V_{1}, V_{2}, \ldots, V_{k}$, called color classes, such that the endpoints of any edge lie in different color classes. The GCP consists of finding the minimum number $k$ such that $G$ admits a $k$-coloring, called the chromatic number of $G$ and denoted by $\chi(G)$.

Some applications impose additional restrictions. For instance, in scheduling problems, it may be required to ensure the uniformity of the distribution of workload employees. Suppose that a set of tasks must be assigned to a set of workers so that pairs of tasks may conflict each other, meaning that they should 
not be assigned to the same worker. The problem is modeled by building a graph containing a vertex for every task and an edge for every conflicting pair of tasks. Workers are represented by colors. Then, in order for a coloring of this graph to represent a valid assignment of tasks to workers, the same number of tasks must be assigned to each worker. Since this is impossible when the number of tasks is not divisible by the number of workers, one can ask for the number of tasks assigned to two arbitrary workers can not differ by more than one. It is called equity constraint and the resulting problem is called Equitable Coloring Problem (ECP).

ECP was introduced in [1], motivated by an application concerning garbage collection [2]. Other applications of the ECP concern load balancing problems in multiprocessor machines [3] and results in probability theory [4]. An introduction to ECP and some basic results are provided in [5].

Formally, an equitable $k$-coloring (or just $k$-eqcol) of a graph $G$ is a $k$-coloring satisfying the equity constraint, i.e. the size of two color classes can not differ by more than one unit. The equitable chromatic number of $G, \chi_{e q}(G)$, is the minimum $k$ for which $G$ admits a $k$-eqcol. The ECP consists of finding $\chi_{e q}(G)$ which is an $\mathcal{N} \mathcal{P}$-Hard problem [5].

There exist some differences between GCP and ECP that make the latter harder to solve. It is known that the chromatic number of a graph is greater than or equal to the chromatic number of any of its induced subgraphs. Unfortunately, in the case of ECP, this property does not hold. For instance, if $G$ is the graph shown in Figure 1, by deleting $v_{5}$ from $G, \chi_{e q}(G)$ increases from 2 to 3.

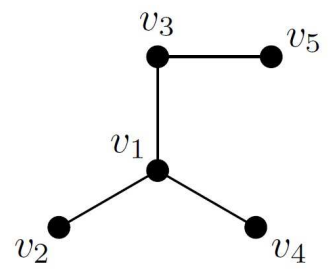

Fig. 1.

As far as we know, there are few approximate and exact algorithms available in the literature related to ECP.

It was proved that, for any graph $G, \Delta(G)+1$ is an upper bound of $\chi_{e q}(G)$ [6], where $\Delta(G)$ is the maximum degree of vertices in $G$. Based on this fact, a polynomial time algorithm for obtaining a $k$-eqcol of a graph $G$ with $k \geq \Delta(G)+1$ is described in [7].

Two constructive heuristics called NaIVE and SubGraph are given in [5] to generate greedily an equitable coloring of a graph. There also exist heuristic algorithms for constructing colorings that are "nearly" equitable $[8,9]$, making 
emphasis on achieving a small difference between the sizes of the biggest class and the smallest one, although the equity constraint still might be violated.

The authors of [10] propose a tabu search heuristic to initialize an exact algorithm that solves ECP via Integer Linear Programming (ILP) techniques. Other exact algorithms for solving ECP are given in [11] and [12]. The first one also uses IPL techinques and the second one is based on a DSATUR enumeration scheme.

In this work, we propose a new heuristic based on the dynamic TABUCOL version of Galinier and Hao [13], one of the best tabu search algorithms for GCP [14]. Then, computational experiments are carried out in order to find the best combination of parameters involved in the dynamic tenure of our heuristic and to show the good performance of it over known benchmark instances.

The paper is organized as follows. In Section 2, we present TABUCOL and the dynamic variant of Galinier and Hao. In Section 3, we give our variant for ECP which we call TABUEQCoL. Finally, in Section 4 we report computational experiences and conclusions.

\section{$2 \quad$ TabuCol and its variants}

Tabu search is a metaheuristic method proposed by Glover [15] that guides a local search algorithm equipped with additional mechanisms that prevent from visiting a solution twice and getting stuck in a local optimum.

Let $S$ be the solution space of the problem and $f: S \rightarrow \mathbb{R}$ be the objective function. The goal is to obtain a solution $s \in S$ such that $f(s)$ is minimum.

For each solution $s \in S$, consider a neighborhood $N(s) \subset S$ with two desirable (but not exclusionary) properties: 1) two solutions $s$ and $s^{\prime}$ are neighbors when it is easy (from the computational point of view) to obtain $s^{\prime}$ from $s$, and to obtain $f\left(s^{\prime}\right)$ from $f(s)$ (for instance, in constant time), and 2) for any $s, s^{\prime} \in S$, there exists a path $s=s_{1}, s_{2}, \ldots, s_{m}=s^{\prime}$ such that $s_{i+1} \in N\left(s_{i}\right)$ for $i=1, \ldots, m-1$.

In general, neighbor solutions are very similar in some sense, and the difference between them can be seen as features that both solutions do not share. Consider a set of features $P$ and a set $R \subset S \times P$ such that $(s, p) \in R$ if solution $s$ presents a feature $p$.

Starting from an initial solution $s_{0} \in S$, tabu search consists of generating a sequence of solutions $s_{1}, s_{2}, \ldots$ such that $s_{i+1}=\arg \min _{s \in N^{\prime}\left(s_{i}\right)} f(s)$, where $N^{\prime}\left(s_{i}\right)$ is a subset of $N\left(s_{i}\right)$ described below. In each iteration of this algorithm, a movement from $s_{i}$ to $s_{i+1}$ is performed and some feature of $s_{i}$ is stored in a tabu list $L \subset P$. This list indicates whether a movement is allowed or forbidden: a solution $s$ can be reached in the future only if $s$ does not present any feature from $L$ (this rule avoids from visiting a solution previously visited), except when $s$ is better than the best solution found so far. This exception is called aspiration and the aspiration criterion is usually to check if the objective value of $s$ is less than the value of currently-known best solution. Now, the set of allowed movements from $s_{i}, N^{\prime}\left(s_{i}\right)$, is defined as

$$
N^{\prime}(s)=\left\{s^{\prime} \in N(s): f\left(s^{\prime}\right)<f\left(s^{*}\right) \vee\left(s^{\prime}, p\right) \notin R \quad \forall p \in L\right\},
$$


where $s^{*}$ is the best solution found so far.

However, after several iterations, old features are no longer needed and it is better to remove them from the tabu list. This mechanism is usually implemented by assigning a "time of live" to each feature of the tabu list. Consider live $: L \rightarrow$ $\mathbb{Z}$ and let live $(p)$ be the number of remaining iterations that $p$ belongs to $L$. When a new feature $p$ is inserted into $L$, live $(p)$ is assigned a value referred to as tabu tenure $t$. Then, in each iteration, the value of live $(p)$ is decreased by one unit until it reachs zero and $p$ is removed from $L$. Above, we sketch a generic tabu search algorithm.

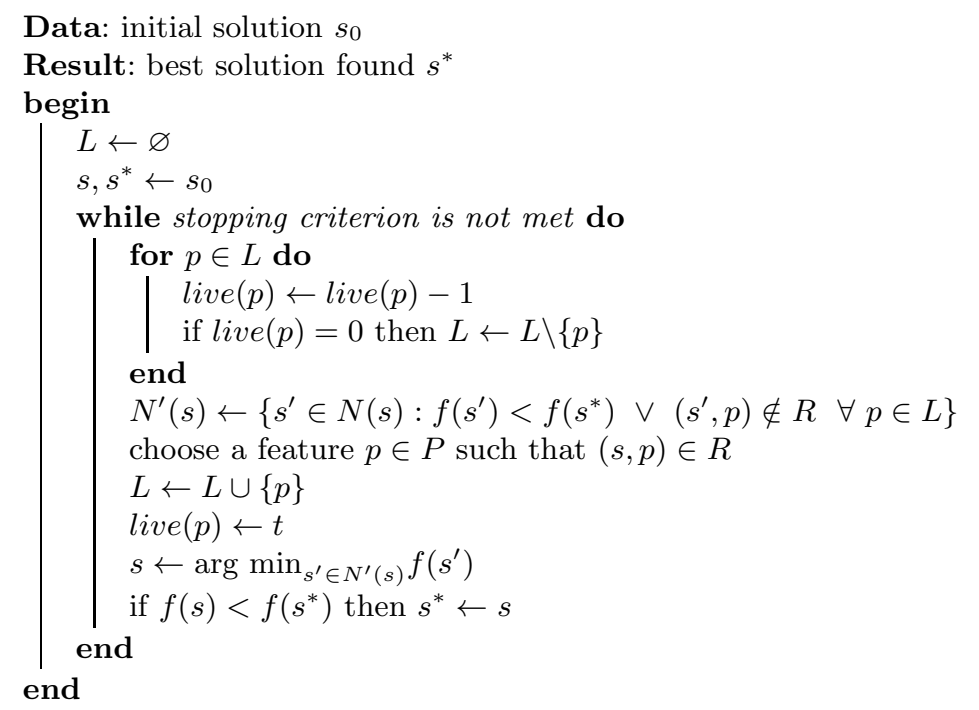

Algoritmo 1: TABUSEARCH

In order to implement a tabu search algorithm, some decisions must be taken: neighborhood of a solution, features of a solution, stopping criterion, how to choose the feature $p$ to be stored in the tabu list and how to compute the tabu tenure $t$. In particular, the value of tabu tenure directly impacts diversification of the algorithm. A tabu search with low tenures behaves as a standard local search, where it frequently get trapped in local minima. On the other hand, a tabu search with high tenures tends to wander across solution space without converging towards the optimal solution.

TABUCoL, the first tabu search algorithm designed for solving GCP, was proposed by Hertz and de Werra [16]. For a given graph $G=(V, E)$ and number $k \in\{1, \ldots, n\}$, where $n=|V|$, the goal of this algorithm is to find a $k$-coloring of $G$. In order to obtain a coloring that uses as few colors as possible, it is usual to embed TABUCOL in a routine that, once a $k$-coloring is found, the algorithm 
can be restarted with $k \leftarrow k-1$ and so on, until some criterion is met. Details of TABUCOL are given below:

- Search space and objective function. A solution $s$ is a partition $\left(V_{1}, V_{2}, \ldots, V_{k}\right)$ of the set of vertices. Let $E\left(V_{i}\right)$ be the set of edges of $G$ with both endpoints in $V_{i}$. The objective function is defined as

$$
f(s)=\sum_{i=1}^{k}\left|E\left(V_{i}\right)\right| .
$$

Clearly, $s$ is a $k$-coloring if and only if $f(s)=0$.

- Stopping criterion. The algorithm stops when $f(s)=0$ or when a maximum number of iterations is reached. Sometimes, a time limit is imposed.

- Initial solution. It is generated randomly. A suitable procedure given in [17] is the following. Start with empty sets $V_{1}, V_{2}, \ldots, V_{k}$ and, at each step, choose a non-considered vertex $v$ randomly and put it into $V_{i}$ with the smallest possible $i$ such that $E\left(V_{i}\right)$ is not incremented. If it is not possible, choose a random number $j \in\{1, \ldots, k\}$ and put $v$ into $V_{j}$.

- Set of features. It is $P=V \times\{1, \ldots, k\}$. A solution $s$ presents a feature $(v, i)$ if and only if $v \in V_{i}$, i.e. if $v$ is assigned color $i$.

- Neighborhood of a solution. Let $C(s)$ be the set of conflicting vertices of a solution $s$, i.e.

$C(s)=\left\{v \in V: v\right.$ is incident in some edge of $\left.E\left(V_{1}\right) \cup E\left(V_{2}\right) \cup \ldots \cup E\left(V_{k}\right)\right\}$.

From a solution $s=\left(V_{1}, V_{2}, \ldots, V_{k}\right)$, a neighbor $s^{\prime}=\left(V_{1}^{\prime}, V_{2}^{\prime}, \ldots, V_{k}^{\prime}\right)$ is generated as follows. Choose a conflicting vertex $v \in C(s)$. Let $i$ be the color of $v$ in $s$. Next, choose a color $j \in\{1, \ldots, k\} \backslash\{i\}$ and set

$$
V_{j}^{\prime}=V_{j} \cup\{v\}, \quad V_{i}^{\prime}=V_{i} \backslash\{v\}, \quad V_{l}^{\prime}=V_{l} \quad \forall l \in\{1, \ldots, k\} \backslash\{i, j\} .
$$

In other words, $s^{\prime}$ is a copy of $s$ except that $v$ is moved from class color $V_{i}$ to $V_{j}$. We denote such operation with $s^{\prime}=s(i \stackrel{v}{\rightarrow} j)$. Note that objective value can be computed in linear time from $f(s)$ :

$$
f\left(s^{\prime}\right)=f(s)+\left|\left\{v w \in E: w \in V_{j}\right\}\right|-\left|\left\{v w \in E: w \in V_{i}\right\}\right| .
$$

Note also that searching all the neighbors of $s$ requires exploring $(k-1)|C(s)|$ solutions. Original TABUCOL only explores a random subset of $N(s)$ while newer versions explore $N(s)$ completely.

- Selection of feature to add in the tabu list. Once a movement from $s$ to $s(i \stackrel{v}{\rightarrow} j)$ is performed, $p=(v, i)$ is stored on tabu list and live $(p)$ is set to a fixed tabu tenure $t=7$.

Later, Galinier and Hao [13] improved TABUCOL by using a dynamic tabu tenure that depends on the quality of the current solution, encouraging diversification of the search when solution is far from optimal. They proposed to assign a tenure of $t=\alpha|C(s)|+\operatorname{Random}(\beta)$ where $\operatorname{Random}(\beta)$ returns an integer randomly chosen from $\{0, \ldots, \beta-1\}$ with uniform distribution. Based on experimentation, they suggest to use $\alpha=0.6$ and $\beta=10$. Other variants of TABUCOL are discussed in $[14,17]$. 


\section{TabuEqCol: A tabu search for ECP}

In this section, we present a new tabu search algorthm for ECP based on TABUCoL with dynamic tabu tenure, which we call TABUEQCoL.

Given a graph $G=(V, E)$ and a number $k \in\{1, \ldots, n\}$, where $n=|V|$, the goal of TABUEQCOL is to find a $k$-eqcol of $G$.

Solution space consists of partitions of $V$ into $k$ sets $V_{1}, V_{2}, \ldots, V_{k}$ such that they satisfy the equity constraint, i.e. for any pair of classes $V_{i}$ and $V_{j},|| V_{i} \mid-$ $\left|V_{j}\right| \mid \leq 1$. Objective function $f$ is the same as in TABUCOL, so any solution $s$ such that $f(s)=0$ is indeed an equitable coloring. Also, set of features $P$ is the same as in TABUCOL.

Stopping criterion depends on the experiment carried out. Usually, a time limit is imposed.

Let $s \in S$. Denote $W^{+}(s)=\left\{i:\left|V_{i}\right|=\lfloor n / k\rfloor+1\right\}$ and $W^{-}(s)=\{i$ : $\left.\left|V_{i}\right|=\lfloor n / k\rfloor\right\}$, where $V_{i}$ are the color classes of $s$. Since $s$ satisfies the equity constraint, we have that $W^{+}(s)$ and $W^{-}(s)$ determine a partition of $\{1, \ldots, k\}$ and, in particular, $\left|W^{+}(s)\right|=r$ where $r=n-k\lfloor n / k\rfloor$. From now on, we just write $W^{+}$and $W^{-}$. These sets will be useful in the development of the algorithm.

We propose two greedy procedures for generating initial solution $s_{0}$.

Procedure 1. Start with empty sets $V_{1}, V_{2}, \ldots, V_{k}$ and an integer $\tilde{r} \leftarrow 0$ (this value will have the cardinal of $\left.W^{+}\right)$. At each step, define set $I=\left\{i:\left|V_{i}\right| \leq M-1\right\}$, where $M$ is the maximum allowable size of a class:

$$
M= \begin{cases}\lfloor n / k\rfloor+1, & \text { if } \tilde{r}<r \\ \lfloor n / k\rfloor, & \text { if } \tilde{r}=r\end{cases}
$$

(once we already have $r$ class of size $\lfloor n / k\rfloor+1$, the size of the remaining classes must not exceed $\lfloor n / k\rfloor$ ). Then, choose a non-considered vertex $v$ randomly and put it into a class $V_{i}$ such that $i \in I$ is the smallest possible and $E\left(V_{i}\right)$ is not incremented. If it is not possible, $i$ is chosen ramdonly from $I$. To keep $\tilde{r}$ up to date, each time a vertex is added to a set $V_{i}$ such that $\left|V_{i}\right|=\lfloor n / k\rfloor, \tilde{r}$ is incremented by one unit.

The previous procedure works fine for generating initial solutions from scratch. However, at this point it is common to know a $(k+1)$-eqcol (i.e. in the cases where we previously ran tabu search with $k+1$ and reached an equitable coloring) and we can exploit this coloring in order to improve the quality of the initial solution as follows.

Procedure 2. Let $\mathfrak{p}:\{1, \ldots, k+1\} \rightarrow\{1, \ldots, k+1\}$ be a bijective function (i.e. a random permutation) and let $V_{1}^{*}, V_{2}^{*}, \ldots, V_{k}^{*}, V_{k+1}^{*}$ be the color classes of the known $(k+1)$-eqcol. Set $V_{i}=V_{\mathfrak{p}(i)}^{*}$ for all $i \in\{1, \ldots, k\}$, and $\tilde{r}=\left|W^{+}\right|$. Then, run Procedure 1 to assign a color to the remaining vertices which are those belonging to $V_{\mathfrak{p}(k+1)}^{*}$. 
Regarding neighborhood of a solution $s \in S$ notice that, if $n$ does not divide $k, W^{+} \neq \varnothing$ and it is possible to move a vertex from a class of $W^{+}$to $W^{-}$, keeping equity. That is, for all $v \in \cup_{i \in W^{+}} V_{i}$ and all $j \in W^{-}$, we have $s(i \stackrel{v}{\rightarrow} j) \in S$. However, the number of allowed movements is rather limited when $r$ is very low (for instance, $r=1)$ or very high $(r=k-1)$, so we need to add supplementary movements. Swapping the colors of two vertices simultaneously seems to work fine and as well can be used when $n$ divides $k$.

From a solution $s=\left(V_{1}, V_{2}, \ldots, V_{k}\right)$, a neighbor $s^{\prime}=\left(V_{1}^{\prime}, V_{2}^{\prime}, \ldots, V_{k}^{\prime}\right)$ is generated with two schemes:

- 1-move (only applicable when $n$ does not divide $k$ ). Choose a conflicting vertex $v \in C(s) \cap\left(\cup_{i \in W^{+}} V_{i}\right)$. Let $i$ be the color of $v$ in $s$. Next, choose a color $j \in W^{-}$. We have $s^{\prime}=s(i \stackrel{v}{\rightarrow} j)$. Searching all the neighbors of $s$ with this scheme requires exploring $(k-r)\left|C(s) \cap\left(\cup_{i \in W^{+}} V_{i}\right)\right|$ solutions.

- 2-exchange. Choose a conflicting vertex $v \in C(s)$. Let $i$ be the color of $v$ in $s$. Next, choose another vertex $u$ such that either $i<j$ or $u \notin C(s)$, where $j$ is the color of $u$ in $s$ (the condition imposed to $u$ prevents from evaluating 2-exchange on $u$ and $v$ twice). Then, set

$V_{j}^{\prime}=\left(V_{j} \backslash\{u\}\right) \cup\{v\}, \quad V_{i}^{\prime}=\left(V_{i} \backslash\{v\}\right) \cup\{u\}, \quad V_{l}^{\prime}=V_{l} \quad \forall l \in\{1, \ldots, k\} \backslash\{i, j\}$.

Note that objective value can be computed in linear time from $f(s)$ :

$$
\begin{aligned}
f\left(s^{\prime}\right)=f(s)+\mid\{u w & \left.\in E: w \in V_{i} \backslash\{v\}\right\}|-|\left\{u w \in E: w \in V_{j}\right\} \mid \\
& +\left|\left\{v w \in E: w \in V_{j} \backslash\{u\}\right\}\right|-\left|\left\{v w \in E: w \in V_{i}\right\}\right| .
\end{aligned}
$$

Searching all the neighbors of $s$ with this scheme requires exploring a quadratic number of solutions.

Now, let $s^{\prime}$ be the next solution in the sucession; $s^{\prime}$ is obtained by applying either 1-move or 2-exchange to $s$, where vertex $v \in V_{i}$ in $s$ and $v \notin V_{i}^{\prime}$ in $s^{\prime}$. In both schemes, $p=(v, i)$ is stored on tabu list and live $(p)$ is set to a dynamic tabu tenure $t=\alpha|C(s)|+\operatorname{Random}(\beta)$ where $\alpha$ and $\beta$ are parameters to be determined empirically. This is one of the purposes of the next section.

\section{Computational experiments and conclusions}

This section is devoted to perform and analyze computational experiments. They were carried out on an Intel i5 CPU 750@2.67Ghz with Ubuntu Linux O.S. and Intel C++ Compiler. We considered graphs from [18], which are benchmark instances difficult to color.

First, we test different combinations of values for parameters $\alpha$ and $\beta$ from the dynamic tabu tenure in order to determine the combination that makes TABUEQCOL perform better. Then, we report the behaviour of TABUEQCOL over known instances by using the best combination previously found. We also compare its performance against tabu search algorithm given in [10]. 


\section{Tuning parameters}

We run TABUEQCOL over 16 instances with a predetermined value of $k$ and an initial solution $s_{0}$ generated with Procedure 1 . The same initial solution is used in all executions of TABUEQCOL for the same instance.

Results are reported in Table 1. First column is the name of the graph $G$. Second and third columns are the number of vertices and edges of $G$. Fourth and fifth columns are known lower and upper bound of $\chi_{e q}(G)$ (obtained by other means). The remaining columns are the time elapsed in seconds by the execution of TABUEQCOL when a $k$-eqcol is found within the term of 1 hour, for each combination. In the case TABUEQCOL is not able to find a $k$-eqcol, $f\left(s^{*}\right)$ is displayed between braces where $s^{*}$ is the best solution found. Three last rows indicate the sum of objective function $f\left(s^{*}\right)$ over non-solved instances, percentage of instances TABUEQCOL solved successfully and the average time elapsed for these instances to be solved.

For the sake of simplicity, we refer to each combination with a capital letter.

Note that combination $\mathrm{D}$ has the least average time, however it has solved less instances than other combinations and the sum of objective values is also worse. We discard A, B, C, D, E and $\mathrm{H}$ with this criterion. By comparing the three remaining combinations, we have that $\mathrm{G}$ is faster than the other two. Even if we restrict the comparison to those 11 instances the 3 combinations solve simultaneously, we have 807 seconds for F, 562 seconds for G and 730 seconds for $\mathrm{I}$, so $\mathrm{G}$ is still better.

We consider combination $\mathrm{G}(\alpha=0.9$ and $\beta=5)$ for TABUEQCoL.

\section{Testing tabu search heuristic}

For each instance, the following process is performed. First, execute NAIVE algorithm (described in [5]) in order to find an initial equitable coloring $c$ of the current instance. Suppose that $k+1$ is the number of colors of $c$. Then, obtain an initial solution $s_{0}$ of $k$ color classes generated from $c$ with Procedure 2, and run TABUEQCOL with parameters $\alpha=0.9$ and $\beta=5$. If a $k$-eqcol is found, start over the process with $k-1$ color classes by running Procedure 2 and TABUEQCOL again. This process is repeated until 1 hour is elapsed or a $\chi_{e q}$-eqcol is reached, and the best coloring found so far is returned.

In Table 2 we report results over 76 benchmark instances with at least 50 vertices (75 from [18] and one Kneser graph used in [10]). First 5 columns have the name of the graph $G$, number of vertices and edges, and best known lower and upper bound of $\chi_{e q}(G)$. Sixth column displays the number of colors of the initial equitable coloring $c$. Seventh and eighth columns display the value $k$ of the best $k$-eqcol found after 30 seconds of execution of our algorithm and the time elapsed in seconds until such $k$-eqcol is reached. If the coloring is optimal, $k$ is displayed in boldface. Next two columns show the same information after 1 
hour of execution, but if the best coloring is found within the first 30 seconds, these columns are left empty.

Time spent by NAIVE is not considered in the computation. However, NAIVE rarely spent more than $1 \mathrm{sec}$. (and never more than $4 \mathrm{sec}$.).

Last two columns show the same information for the tabu search described in [10]. If such information is not available, these columns are left empty. We recall that the values provided in [10] were computed on a different platform (1.8 Ghz AMD-Athlon with Linux and GNU C++ compiler).

Note that our approach reachs optimality in 29 instances and a gap of one unit between $\chi_{e q}$ and the best solution in 7 instances. In other words, it reachs a gap of at most one unit in roughly a half of the evaluated instances. Note also that TABUEQCOL improves the initial solution given by NAIVE in most cases (precisely, 63 instances).

On those instances the value of the best solution given by tabu search of [10] is known, our algorithm gives the same value or a better one. Despite the difference between platforms, it seems that our approach also runs faster.

An interesting fact is that each execution of TABUEQCOL needs no more than 500000 iterations to reach the best value since the largest number of iterations performed was 493204 and took place when TABUEQCOL found a 18-eqcol of DSJC125.5.

In the same sense, TABUEQCOL needs no more than 30000 iterations in each execution and the overall process needs no more than 30 seconds to reach the best value on 56 instances; justly those ones such that columns 9 and 10 are empty. On these instances, the largest number of iterations performed was 28791 and took place when TABUEQCOL found a 10-eqcol of queen9_9.

\section{Conclusion}

The Equitable Coloring Problem is a variation of the Graph Coloring Problem that naturally arises from several applications where the cardinalities of color classes must be balanced. Just like Graph Coloring, the need to solve ap-

plications associated to this new NP-Hard problem justifies the development of exact and approximate algorithms. On large instances, known exact algorithms are unable to address them and heuristics such as NAIVE delivers poor solutions. Our tabu search heuristic based on TABUCOL has shown to improve these solutions and presented a fairly good performance, even if a limit of 30 seconds is imposed. In addition, an iteration limit of 30000 (for a time limit of 30 seconds) and 500000 (for a time limit of 1 hour) can be imposed in order to save time.

Acknowledgements. This work is partially supported by grants UBACYT 20020100100666, PICT 2010-304, PICT 2011-817, PID-UNR ING416 and PIPCONICET 241. 


\section{References}

1. Meyer W.: Equitable Coloring. Amer. Math. Monthly 80, 920-922 (1973)

2. Tucker A.: Perfect graphs and an application to optimizing municipal services. SIAM Review 15, 585-590 (1973)

3. Das S.K., Finocchi I., Petreschi R.: Conflict-free star-access in parallel memory systems. J. Parallel Distrib. Comput. 66, 1431-1441 (2006)

4. Pemmaraju S.V.: Equitable colorings extend Chernoff-Hoeffding bounds. Approximation, Randomization, and Combinatorial Optimization: Algorithms and Techniques, LNCS vol. 2129, pp. 285-296 (2001)

5. Furmanczyk H., Kubale M.: Equitable coloring of graphs. Graph Colorings, Providence, Rhode Island: American Mathematical Society, 35-53 (2004)

6. Hajnal A., Szemerédi E.: Proof of a conjecture of P. Erdös. Combin. theory and its app. 2, 601-623 (1970)

7. Kierstead H., Kostochka A., Mydlarz M., Szemerédi E.: A fast algorithm for equitable coloring. Combinatorica 30, 217-224 (2010)

8. Brélaz D., Nicolier Y., de Werra D.: Compactness and balancing in scheduling. Math. Methods Oper. Res. 21, 65-73 (1977)

9. Sulong G.B.: Some balanced colouring algorithms for examination timetabling. Jurnal Teknologi 19, 57-63 (1992)

10. Bahiense L., Frota Y., Noronha T.F., Ribeiro C.: A branch-and-cut algorithm for the equitable coloring problem using a formulation by representatives. Discrete Appl. Math. 164(1), 34-46 (2014)

11. Méndez Díaz I., Nasini G., Severín D.: A polyhedral approach for the Equitable Coloring Problem. Discrete Appl. Math. 164(2), 413-426 (2014)

12. Méndez Díaz I., Nasini G., Severín D.: An exact DSatur-based algorithm for the Equitable Coloring Problem. Electron. Notes Discrete Math 44, 281-286 (2013)

13. Galinier P., Hao J-K.: Hybrid evolutionary algorithms for graph coloring. J. Comb. Optim. 3(4), 379-397(1999)

14. Galinier P., Hertz A.: A survey of local search methods for graph coloring. Comput. Oper. Res. 33(9), 2547-2562 (2006).

15. Glover F., McMillan C., Novick B.: Interactive decision software and computer graphics for architectural and space planning. Ann. Oper. Res. 5(3), 557-573 (1985)

16. Hertz A., de Werra D.: Using tabu search techniques for graph coloring. Computing 39(4), 345-351 (1987)

17. Blöchliger I., Zufferey, N.: A graph coloring heuristic using partial solutions and a reactive tabu scheme. Comput. Oper. Res. 35(3), 960-975 (2008)

18. Graph Coloring Benchmark Instances. http://www.cs.hbg.psu.edu/txn131/graphcoloring.html 


\begin{tabular}{|c|c|c|c|c|c|c|c|c|c|c|c|c|c|}
\hline \multirow[t]{2}{*}{ Instance } & \multirow[t]{2}{*}{$|V|$} & \multirow[t]{2}{*}{$|E|$} & \multirow[t]{2}{*}{$\underline{\chi_{e q}}$} & \multirow[t]{2}{*}{$k$} & \multicolumn{3}{|c|}{$\mid \begin{array}{c}\alpha=0.3 \\
\beta=5 \beta=10 \beta=15\end{array}$} & \multicolumn{3}{|c|}{$\begin{array}{c}\alpha=0.6 \\
\beta=5 \beta=10 \beta=15\end{array}$} & \multicolumn{3}{|c|}{$\begin{array}{c}\alpha=0.9 \\
\beta=5 \beta=10 \beta=15\end{array}$} \\
\hline & & & & & $\mathrm{A}$ & $\mathrm{B}$ & $\mathrm{C}$ & $\mathrm{D}$ & $\mathrm{E}$ & $\mathrm{F}$ & $\mathrm{G}$ & $\mathrm{H}$ & I \\
\hline DSJR500.1 & 500 & 3555 & 12 & 12 & $\{3\}$ & 1 & 1 & 1 & 1 & 1 & 1 & 1 & 1 \\
\hline DSJR500. 5 & 500 & 58862 & 120 & 131 & $\{14\}$ & $\{3\}$ & $\{1\}$ & $\{8\}$ & $\{3\}$ & 3242 & $\{5\}$ & $\{3\}$ & $\{1\}$ \\
\hline DSJR500.1c & 500 & 121275 & 126 & 195 & $\{4\}$ & $\{1\}$ & 427 & $\{3\}$ & 78 & 747 & 66 & 8 & 11 \\
\hline DSJC500.1 & 500 & 12458 & 5 & 13 & $\{2\}$ & 55 & 41 & 38 & 63 & 47 & 39 & 83 & 57 \\
\hline DSJC500.5 & 500 & 62624 & 13 & 62 & 61 & 530 & $\{1\}$ & $\{1\}$ & $\{1\}$ & $\{2\}$ & $\{1\}$ & $\{2\}$ & $\{1\}$ \\
\hline DSJC500. 9 & 500 & 112437 & 101 & 148 & $\{1\}$ & 106 & 104 & 94 & 91 & 80 & 100 & 90 & 121 \\
\hline DSJC1000.1 & 1000 & 49629 & 5 & 22 & 767 & 411 & 509 & 551 & 423 & 858 & 710 & 691 & 1059 \\
\hline DSJC1000.5 & 1000 & 249826 & 15 & 112 & 543 & 968 & 623 & 518 & 999 & $\{2\}$ & 1853 & $\{2\}$ & $\{1\}$ \\
\hline DSJC1000.9 & 1000 & 449449 & 126 & 268 & 1850 & 1751 & 1822 & 1926 & 1725 & 1250 & 1808 & 1723 & 983 \\
\hline inithx.i.1 & 864 & 18707 & 54 & 54 & $\{8\}$ & $\{8\}$ & $\{8\}$ & $\{8\}$ & $\{7\}$ & $\{7\}$ & $\{8\}$ & $\{8\}$ & $\{7\}$ \\
\hline latin_square_10 & 900 & 307350 & 90 & 131 & 1182 & 1080 & 1013 & 796 & 782 & 946 & 895 & 1298 & 778 \\
\hline flat300_28_0 & 300 & 21695 & 11 & 37 & 238 & $\{1\}$ & $\{1\}$ & 143 & $\{1\}$ & $\{1\}$ & $\{1\}$ & $\{2\}$ & $\{2\}$ \\
\hline flat1000_76_0 & 1000 & 246708 & 14 & 112 & 228 & 548 & 1255 & 154 & 600 & 1681 & 245 & 780 & 3298 \\
\hline abb313GPIA & 1557 & 53356 & 8 & 9 & $\{27\}$ & $\{44\}$ & $\{15\}$ & $\{2\}$ & $\{10\}$ & 2801 & 1796 & $\{1\}$ & 1304 \\
\hline qg. order 40 & 1600 & 62400 & 40 & 40 & 26 & 31 & 17 & 25 & 26 & 20 & 24 & 25 & 26 \\
\hline wap01a & 2368 & 110871 & 41 & 47 & $\{21\}$ & 477 & 501 & $\{6\}$ & 451 & 446 & 499 & 744 & 397 \\
\hline Sum of ob & 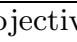 & ve value & & & 80 & 57 & 26 & 28 & 22 & 12 & 15 & 18 & 12 \\
\hline & & & & & $50 \%$ & $69 \%$ & $69 \%$ & $63 \%$ & $69 \%$ & $75 \%$ & $75 \%$ & $63 \%$ & $69 \%$ \\
\hline Avers & age $\mathrm{T}$ & Time & & & 612 & 542 & 574 & 425 & 476 & 1010 & 670 & 544 & 730 \\
\hline
\end{tabular}

Table 1. Execution of TABUEQCOL with different combination of values 


\begin{tabular}{|c|c|c|c|c|c|c|c|c|c|c|c|}
\hline Instance & $|V|$ & $|E|$ & $\chi e q$ & $\overline{\chi e q}$ & Naive & $\leq 3$ & $\begin{array}{r}0 \text { sec. } \\
\text { Time }\end{array}$ & $\leq 1$ & $\begin{array}{c}\text { hour } \\
\text { Time }\end{array}$ & $k$ & $\begin{array}{l}\text { 10] } \\
\text { Time }\end{array}$ \\
\hline miles750 & 128 & 2113 & 31 & 31 & 33 & 31 & 0.0 & & & 35 & 13 \\
\hline miles 1000 & 128 & 3216 & 42 & 42 & 47 & 43 & 0.1 & & & 49 & 13 \\
\hline miles 1500 & 128 & 5198 & 73 & 73 & 74 & 73 & 0.0 & & & 77 & 13 \\
\hline zeroin.i.1 & 211 & 4100 & 49 & 49 & 51 & 51 & 0.0 & & & 74 & 22 \\
\hline zeroin.i.2 & 211 & 3541 & 36 & 36 & 51 & 51 & 0.0 & & & 95 & 22 \\
\hline zeroin.1.3 & 206 & 3540 & 36 & 36 & 49 & 49 & 0.0 & & & 97 & 21 \\
\hline queen8_8 & 64 & 728 & 9 & 9 & 18 & 9 & 1.2 & & & 10 & 7 \\
\hline jean & 80 & 254 & 10 & 10 & 10 & 10 & 0.0 & & & 10 & 3 \\
\hline anna & 138 & 493 & 11 & 11 & 11 & 11 & 0.0 & & & 13 & 14 \\
\hline david & 87 & 406 & 30 & 30 & 40 & 30 & 0.0 & & & 30 & 9 \\
\hline games 120 & 120 & 638 & 9 & 9 & 9 & 9 & 0.0 & & & 11 & 6 \\
\hline kneser9_4 & 126 & 315 & 3 & 3 & 4 & 3 & 0.0 & & & 6 & 2 \\
\hline 2-FullIns_3 & 52 & 201 & 5 & 5 & 9 & 5 & 0.0 & & & 8 & 1 \\
\hline 3-Fullins_3 & 80 & 346 & 6 & 6 & 7 & 6 & 0.0 & & & 9 & 2 \\
\hline 4-FullIns_3 & 114 & 541 & 7 & 7 & 12 & 7 & 0.1 & & & 11 & 5 \\
\hline 5-FullIns_3 & 154 & 792 & 8 & 8 & 9 & 8 & 0.0 & & & 13 & 8 \\
\hline 2-FullIns_5 & 852 & 12201 & 4 & 7 & 15 & 7 & 2.5 & & & & \\
\hline 3-FullIns_5 & 2030 & 33751 & 5 & 8 & 13 & 8 & 25 & & & & \\
\hline 4-Fullins_4 & 690 & 6650 & 6 & 8 & 14 & 8 & 0.4 & & & & \\
\hline 4-FullIns_5 & 4146 & 77305 & 6 & 9 & 21 & 14 & 20 & 9 & 254 & & \\
\hline 1-Insertions_6 & 607 & 6337 & 3 & 7 & 14 & 7 & 0.2 & & & & \\
\hline 2-Insertions_5 & 597 & 3936 & 3 & 6 & 6 & 6 & 0.0 & & & & \\
\hline 3-Insertions_5 & 1406 & 9695 & 3 & 6 & 8 & 6 & 1.2 & & & & \\
\hline homer & 561 & 1628 & 13 & 13 & 13 & 13 & 0.0 & & & & \\
\hline huck & 74 & 301 & 11 & 11 & 11 & 11 & 0.0 & & & & \\
\hline latin_square_10 & 900 & 307350 & 90 & 130 & 460 & 169 & 30 & 130 & 1301 & & \\
\hline DSJC125.1 & 125 & 736 & 5 & 5 & 8 & 5 & 0.8 & & & & \\
\hline DSJC125.5 & 125 & 3891 & 9 & 18 & 27 & 19 & 0.1 & 18 & 788 & & \\
\hline DSJC125.9 & 125 & 6961 & 42 & 45 & 66 & 45 & 0.4 & & & & \\
\hline DSJC250.1 & 250 & 3218 & 4 & 8 & 13 & 9 & 0.1 & 8 & 32 & & \\
\hline DSJC250. 5 & 250 & 15668 & 11 & 32 & 65 & 33 & 7.2 & 32 & 69 & & \\
\hline DSJC250.9 & 250 & 27897 & 63 & 83 & 136 & 83 & 1.2 & & & & \\
\hline DSJR500.1 & 500 & 3555 & 12 & 12 & 12 & 12 & 0.0 & & & & \\
\hline DSJR500. 5 & 500 & 58862 & 120 & 131 & 135 & 133 & 0.1 & & & & \\
\hline DSJR500.1c & 500 & 121275 & 126 & 195 & 349 & 257 & 0.3 & & & & \\
\hline DSJC500.1 & 500 & 12458 & 5 & 13 & 23 & 14 & 3.5 & 13 & 33 & & \\
\hline DSJC500.5 & 500 & 62624 & 13 & 62 & 128 & 63 & 11 & & & & \\
\hline DSJC500. 9 & 500 & 112437 & 101 & 148 & 284 & 182 & 0.7 & & & & \\
\hline DSJC1000. 1 & 1000 & 49629 & 5 & 22 & 38 & 26 & 26 & 22 & 500 & & \\
\hline DSJC1000.5 & 1000 & 249826 & 15 & 112 & 265 & 128 & 27 & 112 & 2261 & & \\
\hline DSJC1000.9 & 1000 & 449449 & 126 & 268 & 575 & 329 & 20 & & & & \\
\hline flat300_20_0 & 300 & 21375 & 11 & 34 & 81 & 38 & 9.2 & 34 & 463 & & \\
\hline flat $300 \_28 \_0$ & 300 & 21695 & 11 & 36 & 65 & 39 & 3.3 & 36 & 3222 & & \\
\hline flat $1000_{-7} 76_{-} 0$ & 1000 & 246708 & 14 & 112 & 223 & 127 & 24 & 112 & 1572 & & \\
\hline fpsol2.i.1 & 496 & 11654 & 65 & 65 & 85 & 78 & 0.1 & & & & \\
\hline fpsol2.i.2 & 451 & 8691 & 47 & 47 & 62 & 60 & 0.0 & & & & \\
\hline fpsol2.i.3 & 425 & 8688 & 55 & 55 & 80 & 79 & 0.0 & & & & \\
\hline inithx.i.1 & 864 & 18707 & 54 & 54 & 70 & 66 & 0.1 & & & & \\
\hline inithx.i.2 & 645 & 13979 & 30 & 93 & 158 & 93 & 7.2 & & & & \\
\hline le450_15b & 450 & 8169 & 15 & 15 & 17 & 16 & 0.3 & 15 & 107 & & \\
\hline le $450 \_15 d$ & 450 & 16750 & 15 & 16 & 30 & 22 & 9.6 & 16 & 599 & & \\
\hline $1 \mathrm{e} 450 \_25 \mathrm{~b}$ & 450 & 8263 & 25 & 25 & 25 & 25 & 0.0 & & & & \\
\hline le $450 \_25 \mathrm{~d}$ & 450 & 17425 & 25 & 27 & 31 & 27 & 29 & & & & \\
\hline le $450 \_5 \mathrm{~b}$ & 450 & 5734 & 5 & 5 & 12 & 7 & 7.2 & & & & \\
\hline le450_5d & 450 & 9757 & 5 & 8 & 18 & 8 & 15 & & & & \\
\hline mug100_25 & 100 & 166 & 4 & 4 & 4 & 4 & 0.0 & & & & \\
\hline mug88_25 & 88 & 146 & 4 & 4 & 4 & 4 & 0.0 & & & & \\
\hline mulsol.i.1 & 197 & 3925 & 49 & 49 & 63 & 50 & 0.0 & & & & \\
\hline mulsol.i.2 & 188 & 3885 & 31 & 48 & 58 & 48 & 0.1 & & & & \\
\hline myciel6 & 95 & 755 & 7 & 7 & 11 & 7 & 0.0 & & & & \\
\hline myciel7 & 191 & 2360 & 8 & 8 & 12 & 8 & 0.1 & & & & \\
\hline qg. order 40 & 1600 & 62400 & 40 & 40 & 64 & 42 & 22 & 40 & 47 & & \\
\hline qg. order 60 & 3600 & 212400 & 60 & 60 & 64 & 64 & 0.0 & 60 & 267 & & \\
\hline queen8_12 & 96 & 1368 & 12 & 12 & 20 & 12 & 0.1 & & & & \\
\hline queen9_9 & 81 & 1056 & 10 & 10 & 15 & 10 & 9.2 & & & & \\
\hline queen10_10 & 100 & 1470 & 10 & 11 & 18 & \begin{tabular}{|l}
10 \\
12
\end{tabular} & 0.1 & 11 & 143 & & \\
\hline school1 & 385 & 19095 & 15 & 15 & 49 & 15 & 12 & & & & \\
\hline school1_nsh & 352 & 14612 & 14 & 14 & 40 & 14 & 14 & & & & \\
\hline wap01a & 2368 & 110871 & 41 & 46 & 48 & 46 & 15 & & & & \\
\hline wap02a & 2464 & 111742 & 40 & 44 & 49 & 47 & 18 & 44 & 83 & & \\
\hline wap03a & 4730 & 286722 & 40 & 50 & 58 & 57 & 18 & 50 & 464 & & \\
\hline abb313GPIA & 1557 & 53356 & 8 & 9 & 17 & 13 & 28 & 10 & 283 & & \\
\hline ash $331 \mathrm{GPIA}$ & 662 & 4181 & 3 & 4 & 8 & 4 & 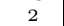 & & & & \\
\hline ash608GPIA & 1216 & 7844 & 3 & 4 & 10 & 4 & 12 & & & & \\
\hline ash958GPIA & 1916 & 12506 & 3 & 4 & 10 & 5 & 11 & 4 & 41 & & \\
\hline will199GPIA & 701 & 6772 & 7 & 7 & 9 & 7 & 2.2 & & & & \\
\hline
\end{tabular}

Table 2. Execution of TABUEQCOL over benchmark instances 